Visentin A. (Orcid ID: 0000-0003-0271-7200)

Autore F. (Orcid ID: 0000-0002-7868-7469)

Tedeschi A. (Orcid ID: 0000-0001-8724-2771)

Laurenti L. (Orcid ID: 0000-0002-4527-4131)

\title{
A SCORING SYSTEM TO PREDICT THE RISK OF ATRIAL FIBRILLATION IN CHRONIC LYMPHOCYTIC LEUKEMIA
}

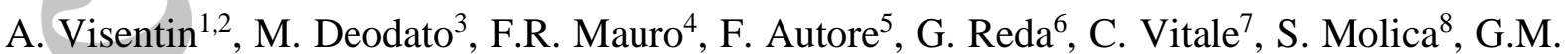

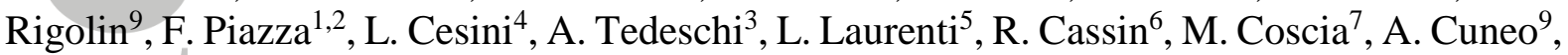
R. Foà ${ }^{4}$, G. Semenzato ${ }^{1,2}$, L. Trentin ${ }^{1,2, *}$

${ }^{1}$ Hematology and Clinical Immunology Unit, Department of Medicine, University of Padua, Italy. ${ }^{2}$ Venetian Institute of Molecular Medicine, Centro di Eccellenza per la Ricerca Biomedica Avanzata, Italy. ${ }^{3}$ Hematology Unit, Niguarda Cancer Center, ASST Grande Ospedale Metropolitano Niguarda, Milan, Italy. ${ }^{4}$ Hematology unit, Department of Translational and Precision Medicine, Sapienza University, Rome, Italy. ${ }^{5}$ Hematology Institute, Fondazione Policlinico Universitario Agostino Gemelli IRCSS, Rome, Italy. ${ }^{6}$ Hematology Unit, Fondazione IRCCS Ca' Granda Ospedale Maggiore, University of Milan, Milan, Italy. ${ }^{7}$ Division of Hematology, A.O.U. Città della Salute e della Scienza di Torino, University of Turin, Turin, Italy. ${ }^{8}$ Department Hematology-Oncology, Azienda Ospedaliera Pugliese-Ciaccio, Catanzaro, Italy. ${ }^{9}$ Hematology section, Department of Medical Sciences, University of Ferrara - Azienda Ospedaliera-Universitaria di Ferrara, Ferrara, Italy.

Running title: risk of atrial fibrillation in CLL

Keywords: chronic lymphocytic leukemia, atrial fibrillation, ibrutinib, comorbidities

* Corresponding author:

Livio Trentin, M.D.

Hematology and Clinical Immunology unit

Department of Medicine

University of Padua

Via Giustiniani 2

35128 Padova, Italy

e-mail: livio.trentin@unipd.it

phone: ++39 049-8212298

Fax: ++390498211970

Summary: 100/100 words

Main text: 1000 words

References: 10

Table: $1 / 1$

Figure: $1 / 1$

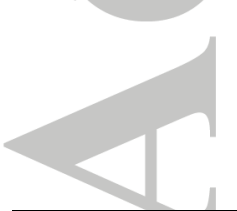

This article has been accepted for publication and undergone full peer review but has not been through the copyediting, typesetting, pagination and proofreading process which may lead to differences between this version and the Version of Record. Please cite this article as doi: 10.1002/hon.2655 


\section{SUMMARY}

Ibrutinib, the first in class of BTK inhibitor, has improved the management of patients with chronic lymphocytic leukemia. Since ibrutinib can cause atrial fibrillation in 6-16\% of cases, it is clinically relevant to identify patients at higher risk to develop atrial fibrillation. Comorbidities associated with a higher risk to develop atrial fibrillation were recapitulated in a scoring system. This model proved to be a valid prognostic tool both in a general chronic lymphocytic leukemia population $(n=860)$ and in an ibrutinib-treated cohort $(n=354)$. Patients with score $\geq 5$ harbored the highest risk of atrial fibrillation and should be carefully monitored during ibrutinib therapy.

\section{MAIN TEXT}

The Bruton's tyrosine kinase (BTK) inhibitor (BTKi) ibrutinib has significantly improved the management of chronic lymphocytic leukemia (CLL). Although ibrutinib is generally well tolerated, it has been associated with atrial fibrillation (AF) in 6-16\% of cases[1]. Most CLL patients are elderly and suffer of several comorbidities[2-3] that may trigger AF regardless of ibrutinib. Since a continuous treatment is required to achieve/maintain the benefit with BTKi, the identification of patients at high-risk of developing AF during ibrutinib is crucial. The purpose we investigated the prevalence and risk factors of AF in a cohort of ibrutinib-naive CLL, in order to define a predictive model for the development of AF and to validate it in a cohort of ibrutinib-treated patients.

We retrospectively analyzed data from 860 CLL patients untreated with or censured at the start ibrutinib, referred to the Padua University Hospital. Diagnosis of CLL fulfilled the iwCLL2008 criteria. Biological markers were analyzed as previously reported[4]. Comorbidities, time to AF (TTAF) and overall survival (OS) were evaluated as described in supplementary methods. Univariate and multivariate Cox models were run to identify independent factors associated with AF. Risk points were obtained based on the half value of hazard ratios (HR). The score for AF was calculated as the sum of each risk point. The model was then evaluated in a cohort of ibrutinib-treated patients.

Among the 860 patients from the Padua hospital, $60 \%$ were male, the median age at diagnosis was 66.8 years, $73 \%$ were Binet A stage at diagnosis and $41 \%$ required at least one treatment. A prior history of AF was present in only 21 patients $(2.4 \%)$ at CLL diagnosis, while, among the remaining 839 patients without a previous history of AF, $47(5.6 \%)$ developed $\mathrm{AF}$ after a median follow-up of 9.4 years (Figure 1A). Comparisons of clinico-biological variables between patients with and without AF are reported in Table1 and S2. The median OS for patients with AF was significantly shorter than that of patients without AF (12 vs 22 years, $\mathrm{p}<0.0001$, Figure 1B).

Based on univariate and multivariate analysis (HRs and confidential intervals [CI] available in Table S1), variables associated with an increased risk of $\mathrm{AF}$ were: age $>65$ years ( $\mathrm{HR}=2.80,1$ point), male gender $(\mathrm{HR}=3.07,1$ point $)$, valvular heart diseases $(\mathrm{HR}=5.71,2$ points), cardiopathy (HR=6.30, 3 points), hypo/hyper-thyroidism ( $\mathrm{HR}=5.61,2$ points), chronic lung diseases ( $\mathrm{HR}=3.69,1$ point), type-2 diabetes mellitus ( $\mathrm{HR}=2.25,1$ point), $\geq \mathrm{G} 3$ infections (HR=2.09, 1 point). A predictive model (AF score) based on the above-mentioned factors identified 4 risk-groups of patients. Although none of the 137 patients at score 0 developed AF, the 545 patients with score of 1-2 had a 10-year TTAF of $6 \%$, while the risk for the 103 and 75 patients at score $3-4$ and $\geq 5$ was $12 \%$ and $29 \%$, respectively ( $p<0.001$, Figure $1 C$ ).

Subsequently, we applied our AF model to a validation cohort of 354 ibrutinib-treated patients referred from 8 Italian hematological centers: $64 \%$ were male, the median age at ibrutinib was 69.7 years, $25 \%$ were treatment-naive, $61 \%$ had an unmutated-IGHV status and $38 \%$ harbored TP53 abnormalities. Forty-two subjects (12\%) developed AF after a median 
observation of 25 months from the start of ibrutinib, with an estimated 2-year TTAF of $12 \%$ (Figure 1D). Median time to AF onset was 10 months. According to the CTCAE grading 38\% were $\geq$ G3 . Patients who developed AF were older and more commonly affected by the comorbidities considered in our scoring system (Table 1). Sixteen patients (4\%) were classified as AF score 0, $218(62 \%)$ score 1-2, $73(21 \%)$ score 3-4 and $46(13 \%)$ at score $\geq 5$. Our model was also able to identify patients at a higher risk of AF during ibrutinib; in fact, the 2-year risk of $\mathrm{AF}$ was $0 \%, 5 \%, 17 \%$ and $40 \%$ for patients with score $0,1-2,3-4$, and $\geq 5$ respectively ( $p<0.001$, Figure 1F). Patients with a score $\geq 5$ have a 20 -fold higher risk of developing AF than the other subjects (HR 19.6, 95\%CI 7-52, p<0.0001). The OS of ibrutinib-treated patients with AF was not inferior to that of patients who did not develop AF (2-year OS 89\% and 82\%, $\mathrm{p}=0.1252$, Figure1E). Only $10 / 44$ (23\%) patients discontinued ibrutinib due to AF. Management of AF was described in supplementary results.

In the Mayo clinic database[5], a prior history of AF was present in 6\% of cases at CLL diagnosis and other $6 \%$ developed it during a median follow-up of 7 years. Age, male gender, valvular heart disease and hypertension were associated with risk of incident AF in multivariate analysis. A predictive model for developing an incident AF that considered these risk-factors stratified patients into 4 subgroups with 10-year rates of AF ranging from $4 \%$ to $33 \%$ [5]. In line with data from the literature[6], in our Italian CLL population a prior history of AF was present in only $2.4 \%$ of patients. While we included chronic lung diseases and severe infections, the other variables were also present in the Shanafelt's scoring system[5]. Hypertension was not statistically significant in our cohort at univariate analysis, and was excluded from multivariate analysis. Comparison of our scoring system with the Shanafelt's model is described in supplementary results and Figure S1.

Although ibrutinib is one of the most commonly used drugs in CLL, the correct management of ibrutinib-induced AF is still of concern[7-10]. In a pooled analysis[1] of 4 clinical trials with ibrutinib, the cumulative incidence of AF was $10 \%$ and most patients (86\%) did not discontinue ibrutinib. Ibrutinib treatment, prior history of AF and age $>65$ years emerged as independent risk factors for the development of AF. More than half of the patients with $\mathrm{AF}$ received anti-coagulant/platelet drugs, $51 \%$ and $2 \%$ reported low-grade and serious bleedings, respectively[1]. In the current work, the cumulative incidence of AF was $12 \%$, most cases did not discontinue ibrutinib and $76 \%$ received anticoagulants without major bleedings nor ischemic events.

In this study, variables associated with an increased risk of developing AF were identified and recapitulated into a scoring system. Taking these data into account, patients with a score $\geq 5$ should be carefully monitored during ibrutinib treatment, or considered for venetoclax/second-generation BTKi.

\section{ACKNOWLEDGEMENTS and FUNDING}

This work was supported by funds from Associazione Italiana per la Ricerca sul Cancro (A.I.R.C.) projects to LT (IG-15397), Gilead fellowship program 2018 to LT, Special Program 'Metastatic disease: the key unmet need in oncology', AIRC 5x1000 (No. 21198) to RF, Fondo di Ateneo per la Ricerca 2016, 2017 of the University of Ferrara to GMR, Fondo di Incentivazione alla Ricerca 2017 of the University of Ferrara to GMR, Ministero dell'Istruzione, dell'Università e della Ricerca PRIN 2015 to AC (2015ZMRFEA). AV received a research fellowship from the University of Padua supported by ONLUS Ricerca per Credere nella Vita (RCV), Padua, Italy. 


\section{AUTHORSHIP CONTRIBUTIONS}

AV designed the study, evaluated patients, performed statistical analysis and wrote the article; MD, FA, CV , FP, LC and RC provided intellectual inputs and evaluated the patients during the follow-up; FRM, SM, GMR, AT, LL, MC, AC, RF, GS, LT evaluated the patients, provided intellectual inputs and reviewed the article.

\section{DISCLOSURE OF CONFLICTS OF INTEREST}

AV received honoraria from Janssen and Abbvie. LT received research funding by Gilead and Janssen, advisory board for Roche, Shire and Abbvie. GMR received research funding by Gilead. FRM advisory board for Janssen, Shire and Abbvie. AC advisory board and speaker bureau for Roche, Abbvie, Gilead and Janssen. GS board member of Abbvie, Roche, Janssen and Celgene. RF advisory board or speaker bureau for Roche, Abbvie, Celgene, Incyte, Amgen, Janssen, Celtrion, Gilead and Novartis.

\section{ETHICAL APPROVAL AND CONSENT TO PARTICIPATE}

This study was approved by the local research ethics committee of Padua hospital and informed consent was obtained from all patients

\section{REFERENCES}

1. Brown JR, Moslehi J, O'Brien S, et al. Characterization of atrial fibrillation adverse events reported in ibrutinib randomized controlled registration trials. Haematologica. 2017; 102:1796-1805.

2. Visentin A, Imbergamo S, Gurrieri C, et al. Major infections, secondary cancers and autoimmune diseases occur in different clinical subsets of chronic lymphocytic leukemia patients. Eur J Cancer. 2017; 72:103-111.

3. Strati P, Parikh SA, Chaffee KG, et al. Relationship between co-morbidities at diagnosis, survival and ultimate cause of death in patients with chronic lymphocytic leukaemia (CLL): a prospective cohort study. Br J Haematol. 2017; 178:394-402.

4. Visentin A, Bonaldi L, Rigolin GM, et al. The combination of complex karyotype subtypes and IGHV mutational status identifies new prognostic and predictive groups in chronic lymphocytic leukemia. Br J Cancer. 2019; Epub. Doi: 10.1038/s41416-019-0502-x

5. Shanafelt TD, Parikh SA, Noseworthy PA, et al. Atrial fibrillation in patients with chronic lymphocytic leukemia (CLL). Leuk Lymphoma. 2017; 58:1630-1639.

6. Zoni-Berisso M, Lercari F, Carazza T, et al. Epidemiology of atrial fibrillation: European perspective. Clin Epidemiol. 2014; 6: 213-220.

7. Mauro FR, Caputo MD, Rosati S, et al. Balancing efficacy and toxicity of targeted agents currently used for the treatment of patients with chronic lymphocytic leukemia. Expert Rev Hematol. 2018 Aug; 11:601-611.

8. Visentin A, Campello E, Scomazzon E, et al. Dabigatran in ibrutinib-treated patients with atrial fibrillation and lymphoproliferative diseases: Experience of 4 cases. Hematol Oncol. 2018 Dec; 36:801-803.

9. Reda G, Fattizzo B, Cassin R, et al. Predictors of atrial fibrillation in ibrutinib-treated CLL patients: a prospective study. J Hematol Oncol. 2018 Jun 11;11:79.

10. Wiczer TE, Levine LB, Brumbaugh J, et al. Cumulative incidence, risk factors, and management of atrial fibrillation in patients receiving ibrutinib. Blood Adv. 2017; 1:17391748. 
Table 1. Clinical-biological characteristics of ibrutinib-naive and ibrutinib-treated patients.

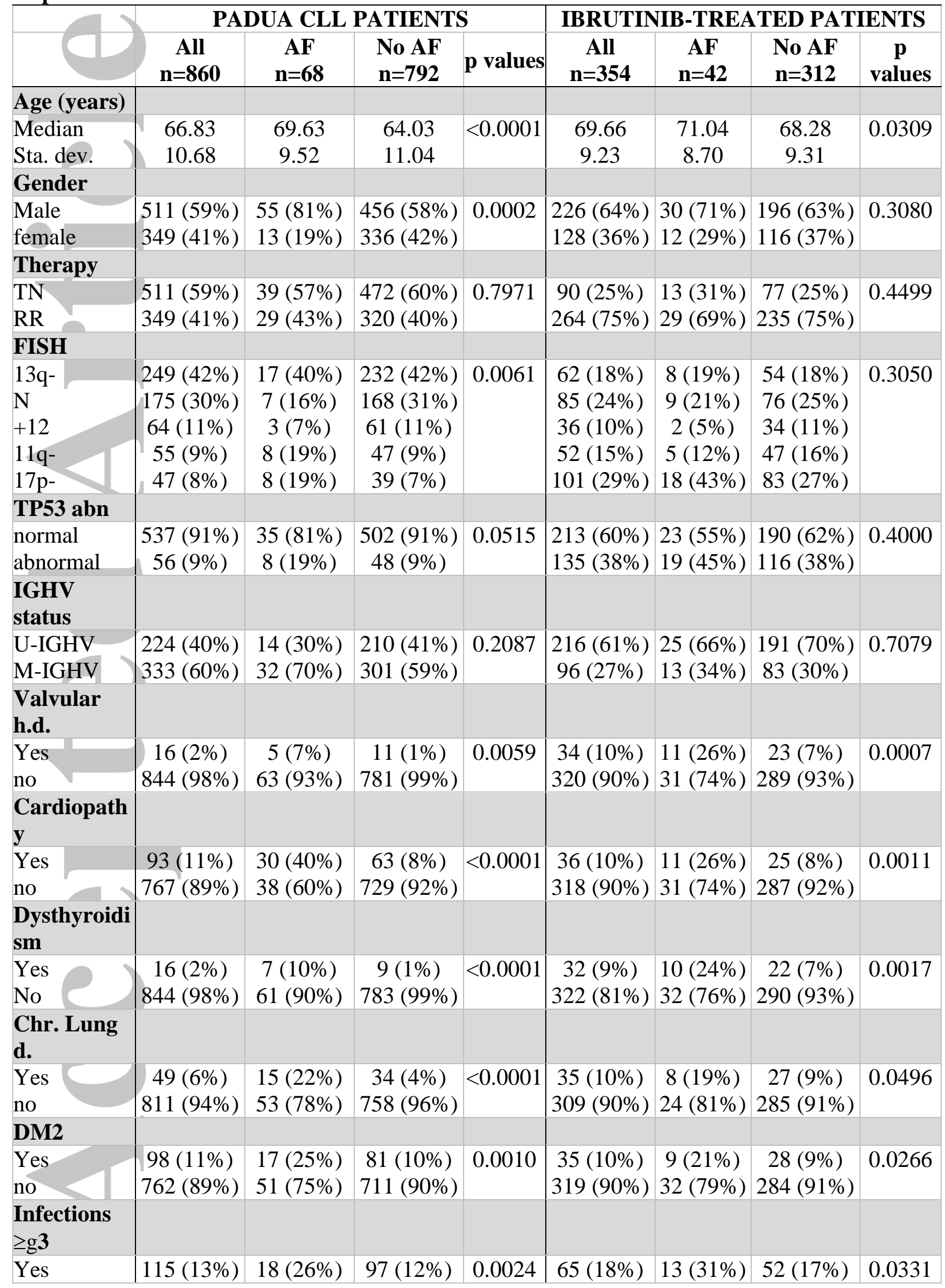

This article is protected by copyright. All rights reserved. 


\begin{tabular}{|c|c|c|c|c|c|c|c|c|}
\hline no & $745(87 \%)$ & $50(74 \%)$ & $695(88 \%)$ & & $289(72 \%)$ & $29(69 \%)$ & $260(83 \%)$ & \\
\hline \multicolumn{9}{|c|}{$\begin{array}{l}\text { Hypertensio } \\
\text { n }\end{array}$} \\
\hline $\begin{array}{l}\text { Yes } \\
\text { No }\end{array}$ & $\begin{array}{l}315(47 \%) \\
545(63 \%)\end{array}$ & $\begin{array}{l}32(47 \%) \\
36(53 \%)\end{array}$ & $\begin{array}{l}283(36 \%) \\
509(64 \%)\end{array}$ & 0.0672 & $\begin{array}{l}- \\
-\end{array}$ & $\begin{array}{l}- \\
-\end{array}$ & $\begin{array}{l}- \\
-\end{array}$ & $\begin{array}{l}- \\
-\end{array}$ \\
\hline \multicolumn{9}{|c|}{$\begin{array}{l}\text { Cholesterol } \\
\text { h. }\end{array}$} \\
\hline $\begin{array}{l}\text { Yes } \\
\text { No }\end{array}$ & $\begin{array}{c}93(11 \%) \\
767(89 \%)\end{array}$ & $\begin{array}{l}12(18 \%) \\
56(82 \%)\end{array}$ & $\begin{array}{c}81(10 \%) \\
711(90 \%)\end{array}$ & 0.0669 & $\begin{array}{l}- \\
-\end{array}$ & $\begin{array}{l}- \\
- \\
\end{array}$ & $\begin{array}{l}- \\
- \\
\end{array}$ & $\begin{array}{l}- \\
- \\
\end{array}$ \\
\hline
\end{tabular}

$A F=$ atrial fibrillation, $T N=$ treatment naive, $R R=$ realpsed or refractory, $U-I G H V=$ unmutated status of IGHV gene (i.e. homology $>98 \%$ from the germiline sequence), M-IGHV $=$ mutated status of IGHV gene, valvular h.d.= moderate to severe valvular heart disease, dysthyroidism = hypo- and hyper-thyroidism, Ch. Lung. $d .=$ chronic lung disease, infections $\geq g 3=$ grade 3-5 infections according to CTCAE, cholesterol $h .=$ cholesterol levels above upper limit values. Clinico-biological variables were analysed using the Mann-Whiney, Fisher's exact or Chi-square tests when appropriated.
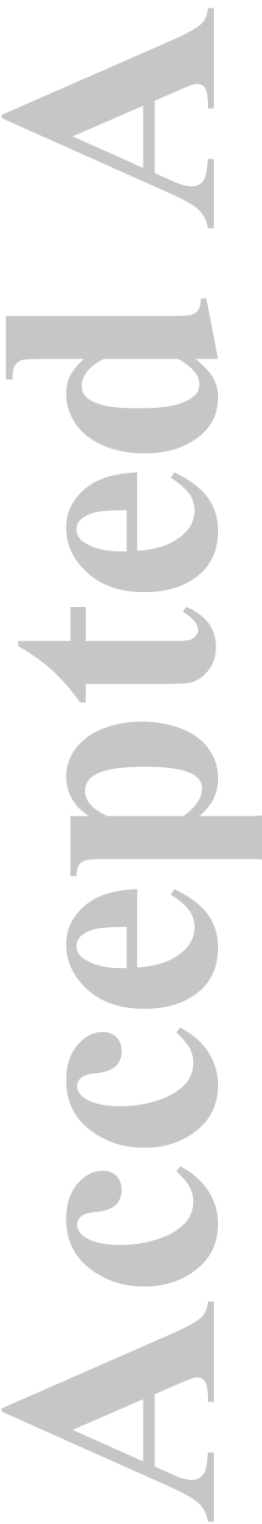
Figure 1

IBRUTINIB-NAIVE PATIENTS
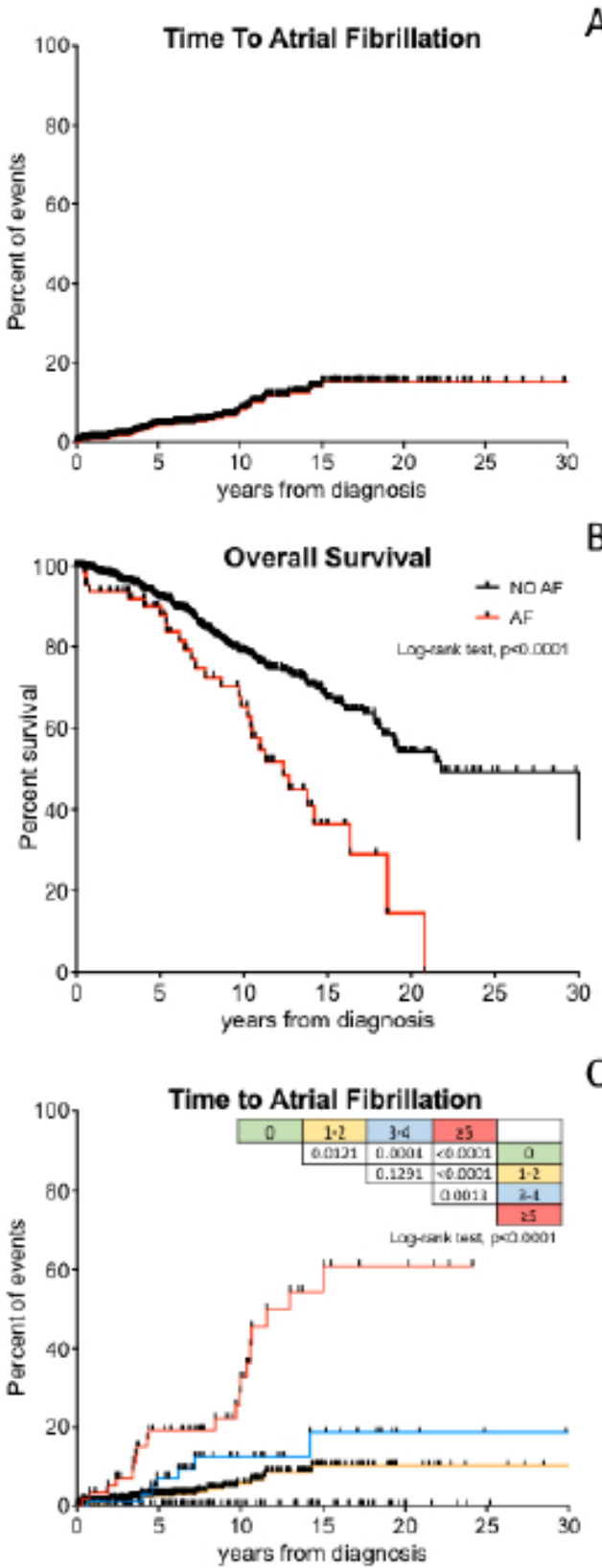

IBRUTINIB-TREATED PATIENTS

A

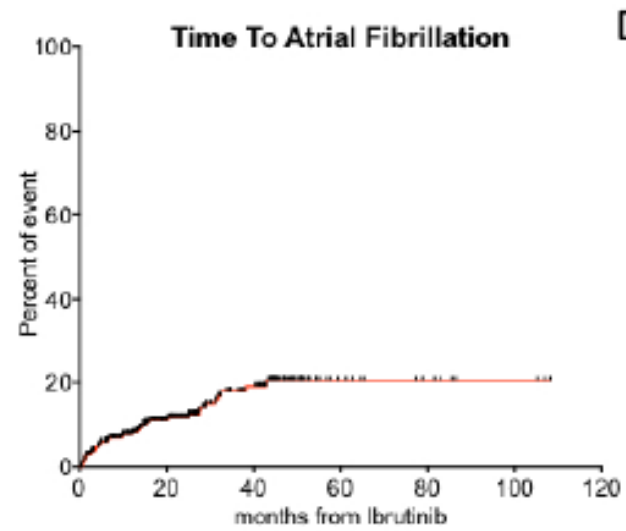

E

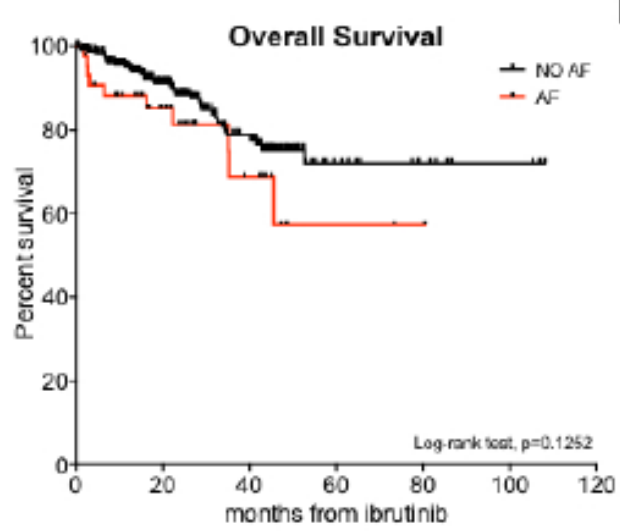

C

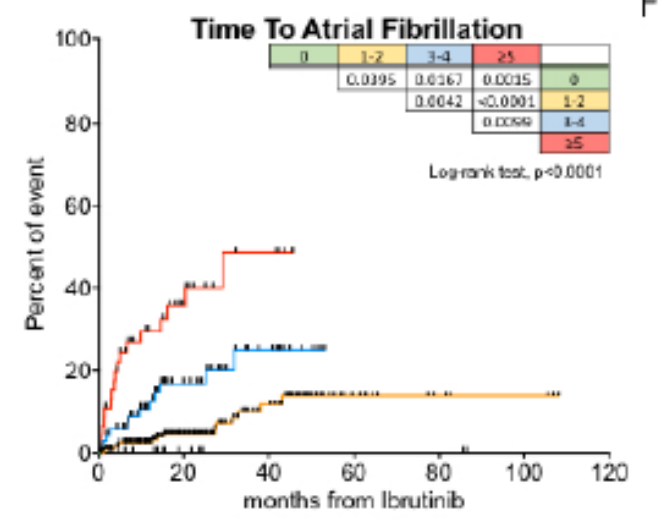

Figure 1. Kaplan-Meier curves of time to atrial fibrillation and overall survival. The left panels show the time to atrial fibrillation (A), overall survival (B) and time to atrial fibrillation according to our proposed scoring system (C) for the ibrutinib-naive patients from the Padua hospital. The right panels show the time to atrial fibrillation (D), overall survival (E) and time to atrial fibrillation according to our proposed scoring system (F) for the cohort of ibrutinib-treated patients. 